\title{
Chief registrar scheme
}

\author{
Authors: Johnny Boylan, Natalie Pink, Tom Baker and Jude Tweedie
}

\begin{abstract}
Aims
The Royal College of Physicians (RCP) Future Hospital Commission Report (2013) ${ }^{1}$ identified the need for new senior leadership roles for doctors in training, to which the chief registrar scheme responded.

\section{Methods}

In 2016 the RCP launched a pilot of the chief registrar scheme. A chief registrar is a senior doctor in training with a minimum of $40 \%$ protected time for leadership development and service improvement within their trust, supported by a bespoke leadership development programme provided by the RCP. Chief registrars deliver a wide range of projects locally in their trusts, focusing on four key areas: service improvement, engagement and morale, education and training and workforce transformation.

To gather evidence and quantify the impact of this scheme, a team from the Health Services Management Centre at the University of Birmingham was commissioned to independently evaluate the impact of the scheme on the chief registrars, the teams they work with, and their wider organisations through monthly surveys and interviews with chief registrars and those they worked with. There was a high degree of variability of both chief registrars and of the contexts in which they worked.
\end{abstract}

\section{Results}

The evaluation highlighted the positive impact that the chief registrar scheme has had on the chief registrars' personal and professional development, the engagement and morale of junior doctors, and their contributions to service improvement. Chief registrar initiatives improved patient flow, led to faster, safer discharge and reduced errors, resulting in a positive impact on downstream costs.

Chief registrars provide a 'bridge' role between junior doctors, senior clinical leaders and managers; engagement improves and morale increases, with evidence of positive consequences for patient safety, patient experience and rota management.

\section{Conclusions}

The evaluation demonstrated the importance of developing leadership and management capacity at doctor in training levels, and demonstrated how the impact of this extends beyond chief registrars to the whole trainee workforce. It demonstrated the benefits to organisations of supporting this important leadership development scheme for trainee doctors: our clinical leaders of tomorrow.

The chief registrar scheme is now in its second year. We are using the learning from the evaluation of the scheme thus far to develop and improve the scheme.

Our goal is to see a chief registrar in every acute hospital, and to have the role fully embedded in the system. The scheme's expansion since the pilot demonstrates substantial and growing interest in developing clinical leadership among trainees across the UK.

\section{Conflict of interest statement}

$\mathrm{NP}$ - project manager

$T B$ - education and training lead

JT and JB - clinical advisers

\section{Reference}

1 Future Hospital Commission. Future hospital: caring for medical patients. A report from the Future Hospital Commission to the Royal College of Physicians. London: RCP, 2013. www.rcplondon.ac.uk/ projects/outputs/future-hospital-commission [Accessed 15 February 2019].

Authors: Royal College of Physicians, London, UK 